\title{
Clinical Application Value and Satisfaction Analysis of Physical Therapy in rehabilitation treatment of cervical spondylosis
}

Jianhong Li

The second community health service center of Balizhuang, Chaoyang District, Beijing, China

Abstract: Objective: To analyze the clinical value and nursing satisfaction of physical therapy in the rehabilitation of patients with cervical spondylosis. Methods: From November 2019 to November 2020, 84 patients with cervical spondylosis were randomly divided into two groups. Group A was given physical therapy, while group B was not given physical therapy. The differences of curative effect, cervical function, quality of life score and nursing satisfaction between the two groups were analyzed. Results: The curative effect of group A was $97.62 \%$ higher than that of group B $83.33 \%, P<0.05$; The score of cervical function in group A $(8.09 \pm 1.75)$ was higher than that in group $\mathrm{B}(8.09 \pm 1.75), P<0.05$; The scores of quality of life in group A were better than those in group $\mathrm{B}(P<0.05)$; The nursing satisfaction of group A was $95.24 \%$, which was better than that of group B 78.57\%, $P<0.05$. Conclusions: Physical therapy intervention for patients with cervical spondylosis can improve nursing satisfaction and cervical function, which can be widely used.

Keywords: Rehabilitation treatment of cervical spondylosis; Physicotherapeutics; Application value; Nursing satisfaction

Publication date: January, 2021

Publication online: 31 January, 2021

"Corresponding author: Jianhong Li,

\section{Introduction} supperpet6210@126.com

The clinical incidence rate of cervical spondylosis is high, typical symptoms are neck shoulder pain and vertigo. With the progress of the disease, pain can involve the upper and lower limbs, restrict normal daily activities, even paralyze, and reduce the quality of daily life of patients. Cervical spondylosis occurs in middle-aged and elderly people, but many patients do not pay enough attention to it. Only a small number of patients go to the hospital in time after cervical spondylosis occurs. Most patients go to the hospital after complicated with pulmonary heart disease, coronary heart disease, diabetes, asthma and other serious complications, which delays the best time for diagnosis and treatment ${ }^{[1]}$. In addition, because patients with cervical spondylosis are often accompanied by other serious underlying diseases, so during the clinical medication treatment, often combined with physical therapy intervention, the effect is good.In this paper, 84 cases of patients with cervical spondylosis rehabilitation treatment to carry out research, explore the application value of physical therapy, the report is as follows.

\section{Material and methods}

\subsection{Information}

The study was carried out in November 2019 and terminated in November 2020. 84 patients with cervical spondylosis were randomly divided into two groups. Group A, male to female ratio 24:18, age 4075 years old, mean $(48.72 \pm 3.29)$ years old, course of disease $1-10$ years, mean $(5.41 \pm 1.25)$ years; In group $\mathrm{B}$, the ratio of male to female was 25 The mean age was $(48.83 \pm 3.34)$ years (range, $42-77$ years). The mean course of disease was $(5.47 \pm 1.31)$ years (range, 2-11 years). CT scan showed calcification, curvature 
change and osteophyte of nuchal ligament; Dizziness, fatigue, numbness and other symptoms were found in all samples; The congenital cervical deformity was removed or the operation was performed. This study was approved by the ethics committee. All patients with cervical spondylosis were informed. The data of 84 patients with cervical spondylosis were compared, $P>0.05$.

\subsection{Treatment}

Group A received physical therapy on the basis of conventional drug intervention. (1) Acupuncture intervention was carried out. The patients were asked to sit down. The points of shousanli, jianwaishu, Fengchi and bilateral Tianzong were selected. The acupuncture method was used to enter the needle and the treatment of lifting, inserting and twisting was carried out. It was appropriate to keep the needle for 15-30 minutes when the patient had a sense of Qi. (2) Cervical traction was performed, and the patient was asked to lie forward $10-15^{\circ}$ for traction treatment. The initial treatment weight was controlled at $4-6 \mathrm{~kg}$, and then the weight was gradually increased according to the muscle condition and the patient's physical recovery ${ }^{[2]}$. (3) Ultrashort wave pulse physiotherapy intervention was performed. The electrotherapy machine was prepared. The large electrode was placed on the back of the patient's neck. According to the patient's condition, whether to load heat or not was determined. Those who need to load micro heat continued to treat for 20 minutes, while those who do not need to load heat continued to treat for 15 minutes. (4) The intermediate frequency electric pulse physiotherapy intervention was carried out to guide the patients to lie on their back and raise their head. It is recommended to raise the head about $5 \mathrm{~cm}$. Then, the electrode plate of the intermediate frequency electric pulse physiotherapy instrument was placed beside the cervical spine to keep the cloth cover in direct contact with the skin. Then, the sandbag was used to squeeze and increase the output current to the maximum tolerable value of the patients, lasting for $20 \mathrm{~min}, 5$ times a week, lasting for 4 weeks $^{[3]}$. (5) For patients with cardiovascular disease, keep the water temperature at $38{ }^{\circ} \mathrm{C}-40{ }^{\circ} \mathrm{C}$. (6) First of all, the nurses should guide the patients to take the correct posture, that is, separate the legs and shoulder width, and let the shoulders droop;Secondly, guide the patient to take a deep breath correctly, then gently raise the head, sink the shoulders, tighten the muscles behind the neck, lasting for 3 seconds, repeated exercise should be tolerated by the patient;Guide the patient to lean his head to one side and the top of his head to the other side, and then guide the patient to stand upright, use the head to write the character of rice, and extend the head to the maximum extent ${ }^{[4]}$.

Group B was given conventional symptomatic drug treatment, including anti-inflammatory and anti swelling drugs, drugs to promote cervical blood circulation, etc.

\subsection{Observation indexes}

The patients' signs and symptoms disappeared, and the neck function returned to normal; The signs and symptoms of the patients were improved, and the neck movement function was basically restored to effective; The patient's physical signs and symptoms were not improved, and the neck activity function was not improved. The JOA scale was used to evaluate the function of cervical spine. The high score indicated that the function of cervical spine recovered well. The difference of nursing satisfaction between the two groups was criticized by the selfmade scale of our hospital, and those with more than 90 scores were considered satisfactory; The score of 60-89 was basically satisfied; Those who scored less than 60 were not satisfied ${ }^{[6]}$. SF-36 scale was used to evaluate the differences of quality of life between the two groups, including physiological function, psychological state, social function, physical activity, etc.

\subsection{Statistical study}

SPSS 21.0 was used to calculate the data of patients with cervical spondylosis, $\%,(\bar{x} \pm s)$ were used to count the number of patients with cervical spondylosis, and the changes of measurement indexes before and after treatment were analyzed by $\chi^{2}, \mathrm{t}$ test. $P<0.05$, can be compared and analyzed.

\section{Results}

\subsection{Analyze the difference of curative effect betw- een groups of patients with cervical spondylosis}

The effect of group A was $97.62 \%$ better than that of group B $(83.33 \%)$, the difference was statistically significant $(P<0.05)$. See Table 1 . 
Table 1. Difference analysis of curative effect of patients with cervical spondylosis $(n, \%)$

\begin{tabular}{ccccc}
\hline Group & Remarkable effect & Effective & Invalid & Total effective rate \\
\hline Group A $(n=42)$ & $24(57.14)$ & $17(40.48)$ & $1(2.38)$ & 97.62 \\
Group B $(n=42)$ & $21(50.00)$ & $14(33.33)$ & $7(16.67)$ & 83.33 \\
$\chi^{2}$ & - & - & - & 4.9737 \\
$P$ & - & - & - & $<0.05$ \\
\hline
\end{tabular}

\subsection{Comparison of cervical function score}

The score of cervical function in group A was significantly higher than that in group $\mathrm{B}(P<0.05)$;
Before the nursing intervention, the score of cervical spine function in the two groups was compared, $P>$ 0.05. As shown in Table 2 .

Table 2. Analysis of cervical function score of patients with cervical spondylosis (points, $\bar{x} \pm s$ )

\begin{tabular}{ccc}
\hline Group & Before treatment & After treatment \\
\hline Group A $(n=42)$ & $5.51 \pm 1.64$ & $12.68 \pm 2.24$ \\
Group B $(n=42)$ & $5.53 \pm 1.66$ & $8.09 \pm 1.75$ \\
$t$ & 0.0555 & 10.4648 \\
$P$ & $>0.05$ & $<0.05$ \\
\hline
\end{tabular}

3.3 Quality of life score of patients with cervical significantly higher than those in group B $(P<0.05)$. spondylosis

As shown in Table 3.

The scores of quality of life in group A were

Table 3. Quality of life scores of patients with cervical spondylosis (points, $\bar{x} \pm s$ )

\begin{tabular}{ccccc}
\hline Group & Physiological function & Mentality & Social function & Physical activity \\
\hline Group A $(n=42)$ & $92.78 \pm 5.97$ & $92.85 \pm 5.79$ & $93.64 \pm 6.02$ & $91.28 \pm 6.14$ \\
Group B $(n=42)$ & $81.47 \pm 5.14$ & $82.46 \pm 5.07$ & $79.29 \pm 5.49$ & $83.57 \pm 5.88$ \\
$t$ & 9.3042 & 8.7493 & 11.4145 & 5.8774 \\
$P$ & $<0.05$ & $<0.05$ & $<0.05$ & $<0.05$ \\
\hline
\end{tabular}

3.4 Compare the difference of satisfaction between groups under different treatment schemes

itation treatment were intervened by physical therapy, the treatment satisfaction was $95.24 \%$, compared with

Group A patients with cervical spondylosis rehabil$78.57 \%$ in group B, $P<0.05$. As shown in Table 4 .

Table 4. Difference analysis of treatment satisfaction of patients with cervical spondylosis $(n, \%)$

\begin{tabular}{ccccc}
\hline Group & Satisfied & Basically satisfied & Dissatisfied & Satisfaction \\
\hline Group A $(n=42)$ & $27(64.29)$ & $13(30.95)$ & $2(4.76)$ & 95.24 \\
Group B $(n=42)$ & $22(52.38)$ & $11(26.19)$ & $9(21.43)$ & 78.57 \\
$\chi^{2}$ & - & - & - & 5.1258 \\
$P$ & - & - & - & $<0.05$ \\
\hline
\end{tabular}

\section{Discussion}

In recent years, the risk of cervical spondylosis is increasing year by year, and it is becoming younger. It is mainly related to the excessive learning pressure of teenagers or long-term dependence on electronic products leading to cervical deformation. After the occurrence of cervical spondylosis, patients are often accompanied with dizziness, upper limb numbness, neck and shoulder pain and other symptoms, which can affect the normal life. Therefore, effective rehabilitation treatment for patients with cervical spondylosis is still a hot spot in clinical research ${ }^{[7]}$.
At present, there are many physical therapy programs for patients with cervical spondylosis, including acupuncture, neck massage, cervical traction, neck movement therapy, etc., which have good effect and can improve the symptoms of cervical vertebra in a short time. Physical therapy is non-invasive operation, which has little impact on human body and longterm effect. Long term adherence to physical therapy can play the role of anti-inflammatory, analgesic, exciting muscles and promoting blood circulation In addition, it can improve the immune ability of the body. In addition, physical therapy has low cost, can reduce the economic pressure of patients, combined 
with western medicine symptomatic treatment, can improve the drug effect, promote drug absorption[8]. Combined with the analysis of this study, the efficacy, cervical function score, quality of life score and nursing satisfaction of group A were better than those of group $\mathrm{B}, P<0.05$. It is suggested that physical therapy can improve the prognosis of patients with cervical spondylosis.

In conclusion, physical therapy for patients with cervical spondylosis rehabilitation treatment, can improve the symptoms of neck and shoulder pain, promote the recovery of neck activity function, and then improve the quality of life of patients, with promotion value.

\section{References}

[1] Zhang M, Zhou JJ, Chen J, et al. Efficacy observation of neck rehabilitation gymnastics combined with Mulligan manipulation in the treatment of cervical spondylotic radiculopathy $[\mathrm{J}]$. Chinese Journal of Physical Medicine and Rehabilitation, 2018, 40(9): 686-688.

[2] Yu CJ, Wu WL, Li DB, et al. Comparison of the effect of cervical functional exercise device and exercise therapy in the treatment of cervical spondylotic radiculopathy [J]. Guangxi Medical Journal, 2018, 40(15): 1647-1649.

[3] Yan SJ, Li XQ, Du Y, et al. Effect analysis of conventional massage combined with lower cervical resistance exercise in the treatment of cervical spondylotic disease [J]. Journal of Neck and Lumbar Pain, 2018, 39(4): 96-98.

[4] Jiang TH, Li JB, Wang ZH, et al. Comparative analysis of therapeutic effect of massage combined with lower cervical impedance exercise in the treatment of cervical spondylosis [J]. Journal of Neck and Lumbar Pain, 2018, 39(3): 98-99.

[5] Liu C, Li WQ. Application analysis of acupuncture combined with physiotherapy rehabilitation in the treatment of cervical spondylosis [J].Journal of Hunan University of Traditional Chinese Medicine, 2018(A01): 344-345.

[6] Li SQ, Ou Y. Effect of traction combined with cervical arthropathy on the treatment of cervical radiculopathy $[\mathrm{J}]$. Chinese Journal of Physical Medicine and Rehabilitation, 2018(1): 63-65.

[7] He ZH, Luo GY, Li JQ, et al. Wu Shan's Experience in Differential Treatment of Cervical Spondylotic Radiculopathy Using Lin's Massage Combined with Motion Acupuncture [J]. Chinese Journal of Information on Traditional Chinese Medicine, 2020, v.27;No. 317(12): 121-124. 